\title{
Elternschaft als (neue) Herausforderung für die Psychiatrie
}

\author{
Parenthood as a (New) Challenge for Psychiatric Services
}

Autoren

Institut
Silvia Krumm, Thomas Becker

Klinik für Psychiatrie und Psychotherapie II der Universität Ulm am BKH Günzburg

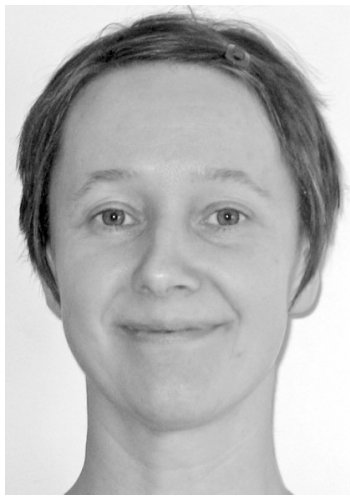

Dr. phil. Silvia Krumm
Bibliografie

DOI http://dx.doi.org/

10.1055/s-0030-1265944

Psychiat Prax 2011; 38: 1-3

(c) Georg Thieme Verlag KG

Stuttgart · New York .

ISSN 0303-4259

\section{Korrespondenzadresse}

\section{Dr. phil. Silvia Krumm}

Klinik für Psychiatrie und Psychotherapie II der Universität Ulm am BKH Günzburg Ludwig-Heilmeyer-Straße 2 89312 Günzburg

Silvia.krumm@bkh-guenzburg. de
Seit geraumer Zeit erfährt das Thema Elternschaft auf gesellschaftlicher Ebene zunehmende Beachtung. Neben einer schier unüberschaubaren Ratgeberliteratur zu Familie und Erziehung lassen sich beinahe täglich Debatten um Reproduktionsziffern, Elterngeld und -zeit oder um die Frage des gesellschaftlichen Werts von Kindern verfolgen. Dieser Diskurs bleibt sicherlich nicht ohne Einfluss auf den individuellen Umgang mit der eigenen (potenziellen) Elternschaft. Kinder zu haben, so die Botschaft an Frauen und Männer, ist eine überaus verantwortungsvolle Aufgabe, die mit hohen Anforderungen an die eigenen Fähigkeiten und Ressourcen für eine optimal ablaufende Elternschaft verknüpft ist. Menschen, die zeitweilig oder dauerhaft von einer psychischen Erkrankung betroffen sind, stehen aber darüber hinaus aufgrund spezifischer reproduktiver Risiken vor besonderen Herausforderungen. Diese Risiken treten bereits beim Umgang mit einem Kinderwunsch hervor. So bergen einige Psychopharmaka teratogene Risiken für den Embryo. Problematisch ist dabei, dass Schädigungen bereits in einer sehr frühen Phase der embryonalen Entwicklung erfolgen können - in einer Phase also, in der die Frauen häufig noch gar nicht wissen, dass sie schwanger sind. Erschwerend kommt hinzu, dass hier eine z.T. eingeschränkte Befundlage besteht $[1,2]$. Das letztlich immer verbleibende Restrisiko bei Weiterführung, Wechsel oder Abbruch der medikamentösen Therapie ist - selbst nach eingehender Beratung durch behandelnde Ärzte - von den betroffenen Frauen selbst zu tragen. Weitere, damit verbundene Risiken betreffen den Krankheitsverlauf der betroffenen Frau: Gerade das abrupte Absetzen eines Medikaments kann ein beträchtliches Rezidivrisiko mit sich bringen. Aber auch im Hinblick auf den weiteren Schwangerschaftsverlauf bestehen Risiken, denn nur ein kleiner Teil der psychiatrisch vorbelasteten Frauen erfährt durch ihre Schwangerschaft eine Stabilisierung ihres psychischen Gesundheitszustan- des. Tatsächlich ist die Annahme eines generell protektiven Effekts einer Schwangerschaft als ein weitverbreiteter „Mythos“ einzuordnen [3]. Auch die Zeit des Wochenbetts geht mit einem erheblichen Rückfallrisiko einher [1]. Schließlich kommen im weiteren Verlauf Risiken für die Entwicklung der Kinder hinzu. Langzeitstudien haben belegt, dass Kinder psychisch kranker Eltern ein erhöhtes Risiko haben, psychopathologische Auffälligkeiten im kognitiven, emotionalen und sozialen Bereich zu entwickeln bzw. selbst an einer psychischen Störung zu erkranken [4,5]. Das erhöhte Risiko für die Kinder geht neben genetischen auch auf Umweltfaktoren zurück, insbesondere in Form einer reduzierten mütterlichen Empathie und Reaktion auf kindliche Signale im Frühkindalter sowie eines unangemessenen Erziehungsstils in späteren Entwicklungsphasen [6].

Schon dieser nur kurz skizzierte Hintergrund macht deutlich, dass Elternschaft für Menschen mit einer psychischen Erkrankung ein kontinuierliches Abwägen zwischen der Berücksichtigung eigener, krankheitsrelevanter Bedürfnisse und den (prospektiven) Bedürfnissen des (ungeborenen) Kindes erfordert. Neben der Problematik im Zusammenhang mit der Medikation während einer Schwangerschaft umfasst dies auch die Einschränkungen in der Erziehungsfähigkeit, die viele Mütter als eine Folge der Medikamenteneinnahme wahrnehmen. Die Befürchtung, entweder die Gesundheit des (ungeborenen) Kindes bzw. dessen psychosoziale Entwicklung oder aber die eigene psychische Stabilität zu gefährden, wird von betroffenen Frauen als belastend erlebt [7-10].

Jenseits aller individuellen Risiken für Mutter und Kind existieren aber auch gesellschaftliche Repräsentationen, die den Umgang mit dem Thema Elternschaft bei Menschen mit einer psychischen Erkrankung moderieren können. Ein weitverbreitetes Stereotyp umfasst die Unfähigkeit psychisch kranker Menschen, ein Kind angemessen zu be- 
treuen und zu erziehen. Elternschaft scheint mit herrschenden Bildern zu psychischen Erkrankungen nur schwer vereinbar. Diese sozialen Repräsentationen werden durch den eingangs erwähnten Diskurs möglicherweise verstärkt. Wie im Rahmen einer kanadischen Studie gezeigt wurde, arbeiten Medien häufig mit Bildern der „gefährlichen“ und „unberechenbaren“ psychisch kranken Mutter, während Berichte über die Schwierigkeiten und Belastungen von Müttern mit psychischen Erkrankungen oder gar über Modelle guter Praxis in der Berichterstattung eher selten sind [11]. Derartige Repräsentationen spiegeln sich in den Stigmatisierungserfahrungen vieler psychisch kranker Mütter wider $[8,9,12,13]$. Irritierend ist dabei der Befund, dass Stigmatisierungen nicht auf den privaten Bereich beschränkt sind, sondern auch dem professionellen Umfeld entstammen [14]. Es finden sich Hinweise darauf, dass betreuende Ärzte zwar nicht direkt von einer Schwangerschaft abraten, allerdings durch entsprechende Äußerungen indirekte Sterilisationsempfehlungen geben und einen geäußerten Kinderwunsch eher ignorieren und damit auch keine Unterstützung anbieten [15]. Bis in die 80erJahre findet sich in fachpsychiatrischen Publikationen - meist einleitend - die generalisierte Annahme, dass eine Schwangerschaft bei psychiatrischen Patientinnen durch eine sichere Verhütungsmethode zu verhindern ist (z.B. [16-20]). Es erstaunt nicht, wenn betroffene Frauen unter diesen Umständen institutionalisierte Hilfsangebote eher zögerlich annehmen [21].

Auch wenn derlei Einstellungen nur noch von marginaler Bedeutung für die heutige psychiatrische Versorgung sein dürften, so ist doch anzunehmen, dass sich die Beschäftigten in einem Spannungsfeld zwischen der Respektierung einer autonomen reproduktiven Entscheidung für (oder gegen) eine Elternschaft einerseits und der Fürsorge gegenüber der betroffenen Person und/ oder dem (ungeborenen) Kind andererseits bewegen. Der professionelle Umgang mit dem Thema Kinderwunsch/Elternschaft ist als ein paradigmatischer Ausdruck des Dilemmas der Psychiatrie einzuordnen, bei dem die Berücksichtigung des Fürsorgeprinzips - sowohl gegenüber der Mutter wie auch gegenüber dem Kind mit einer Verletzung der Patientenautonomie einhergehen kann und vice versa. Die weitgehende Vernachlässigung des Themas Elternschaft im psychiatrischen Fachdiskurs könnte auch eine Reaktion auf die Wahrnehmung dieses ethischen Spannungsfelds sein, das gerade in Deutschland durch die historische Hypothek der systematischen Verhinderung der Fortpflanzung psychisch kranker Menschen - von Zwangssterilisation bis hin zur Tötung - schwer belastet ist. Die Deutsche Gesellschaft für Soziale Psychiatrie begann in den späten 70er-Jahren, auch in Zusammenarbeit mit dem Institut für Zeitgeschichte in München, mit der Aufarbeitung der Geschichte der Psychiatrie im Nationalsozialismus [22]. Die Deutsche Gesellschaft für Psychiatrie, Psychotherapie und Nervenheilkunde (DGPPN) stellt sich dezidiert ihrer Verantwortung, die aus der Beteiligung der Psychiatrie an den Krankenmorden und Zwangssterilisierungen erwachsen ist: „Psychiatrie im Nationalsozialismus“ war eines der Hauptthemen des letztjährigen DGPPN-Kongresses in Berlin. ${ }^{1}$

Tatsächlich nimmt sich die psychiatrische Versorgungsforschung wie auch die Versorgungspraxis der Bedeutung reproduktiver Themen in den Biografien psychisch kranker Menschen erst seit Kurzem an. Während diese Thematik von psychiatrischer Seite lange Zeit auf die Frage einer sicheren Verhütung und auf die Problematisierung der Risiken für Kinder psychisch kranker Eltern

\footnotetext{
${ }^{1}$ Abrufbar im Internet auf der DGPPN-Kongresshomepage http://www. dgppn.de/dgppn-kongress.html (letzter Aufruf am 26.10.2010).
}

beschränkt war, haben insbesondere Arbeiten aus den USA und aus Großbritannien den Blick auf die betroffenen Subjekte - in der Regel Mütter - gelegt, auf die hohe subjektive Bedeutung des Themas Elternschaft wie auf die damit einhergehenden Schwierigkeiten und insbesondere auf die mangelnde soziale und professionelle Unterstützung und Stigmatisierungsphänomene hingewiesen. Dieser Forschungsrichtung ist es zu verdanken, dass sich in der Beschäftigung mit dem Thema Kinder psychisch kranker Eltern der Fokus von der Situation des einzelnen Kindes allmählich auf die gesamte Familie erweitert. Diesem Perspektivenwechsel liegt die wichtige Einsicht zugrunde, dass eine adäquate Unterstützung des Elternteils dem gesamten Familiensystem - den betroffenen Kindern, dem erkrankten Elternteil wie auch dem nicht erkrankten Partner sowie weiteren Angehörigen als wichtige Stütze - zugute kommt. Der ressourcenbetonende Ansatz ist nicht nur dem Wunsch nach wertschätzendem Umgang mit einem biografisch relevanten Thema geschuldet. Er entspricht auch der Befundlage, dass die soziale Entwicklung der Kinder durch ungünstige Sozialisationsbedingungen nicht unbedingt und ausschließlich negativ beeinflusst wird. Vielmehr können sie sich bei einer erfolgreichen Bewältigung durchaus positiv im Sinne einer Ressource aktiver Lebensbewältigung auswirken [23].

Es gehört daher zu den Aufgaben der Psychiatrie, Menschen mit psychischen Erkrankungen auch in ihrer (prospektiven) Elternrolle zu stärken - angefangen von familienorientierten Beratungsangeboten über ambulante bzw. stationäre Eltern-KindProgramme bis hin zur adäquaten Unterstützung in der Trauerarbeit bei Sorgerechtsverlust oder erkrankungsbedingter, unfreiwilliger Kinderlosigkeit. Dies erfordert aber zunächst die Anerkennung der zentralen Rolle, die reproduktive Aspekte im Leben vieler Menschen spielen. Begleitend dazu stellen sich auf theoretischer Ebene Fragen hinsichtlich einer (Neu-)Bestimmung familienpsychiatrischer Konzepte. Mit diesem Schwerpunktheft möchten wir einen Beitrag dazu leisten, dass das Thema Elternschaft noch stärker als bisher seinen Platz in der psychiatrischen Versorgung erhält [24].

\section{Literatur}

1 Rohde A, Schaefer C. Psychopharmakotherapie in Schwangerschaft und Stillzeit. Arzneisicherheit, Beratung, Entscheidungsfindung. Stuttgart: Thieme; 2010

2 Krüger S. Antipsychotika-Therapie und Kinderwunsch. Neurotransmitter 2006; 11: 45-46

3 Einarson A. Introduction: Reproductive mental health. Can J Clin Pharmacol 2009; 16: e1-e5

4 Niemi L, Suvisaari J, Haukka J et al. Cumulative incidence of mental disorders among offspring of mothers with psychotic disorder. Results from the Helsinki High-Risk Study. Br J Psychiatry 2004; 185: 11-17

5 Schubert EW, McNeil TF. Prospective study of adult mental disturbance in offspring of women with psychosis. Arch Gen Psychiatry 2003; 60: 473-480

6 Mattejat F, Remschmidt H. Children of mentally ill parents. Dtsch Arztebl Int 2008; 105: 413-418

7 Krumm S, Kilian R, Becker T. „Ein Kind wäre schon ein Wunsch...“ Psychische Erkrankung und Kinderwunsch aus der subjektiven Sicht betroffener Frauen - Eine qualitative Untersuchung. Psychiat Prax 2010; 37: 134-141

8 Diaz-Caneja A, Johnson S. Views and experiences of severely mentally ill mothers. Soc Psychiatry Psychiatr Epidemiol 2004; 39: 472-482

9 Savvidou I, Vasilis F, Hatzigeleki S et al. Narratives about their children by mothers hospitalized on a psychiatric unit. Fam Process 2003; 42: 391-402

10 Nicholson J, Sweeney E, Geller J. Mothers with mental illness: I. The competing demands of parenting and living with mental illness. Psychiatr Serv 1998; 49: 635-642 
11 Greaves L, Varcoe C, Poole $N$ et al. A motherhood issue: Discourses on mothering under duress. Ontario: National Library of Canada; 2002

12 Chernomas W, Clarke D, Chisholm F. Perspectives of women living with schizophrenia. Psychiatr Serv 2000; 51: 1517-1521

13 Nicholson J, Sweeney E, Geller J. Mothers with mental illness: II. Family relationships and the context of parenting. Psychiatr Serv 1998; 49: 643-649

14 Apfel R, Handel M. Madness and the loss of motherhood. Sexuality, reproduction, and long-term mental illness. Washington: American Psychiatric Press; 1993

15 Sachse L. „Ich bin ganz und richtig“ Therapeutische Begleitung durch Psychose und Mutterschaft. Neumünster: Paranus Verlag; 2000

16 Wignall C, Meredith C. Illegitimate pregnancies in State institutions. Arch Gen Psychiatry 1968; 18: 580-583

17 Ernst K, Ernst C. Familie, Pubertät und Generationsvorgänge in der Anamnese neurotischer Klinikpatientinnen. Arch Psychiat Nervenkr 1969; 212: 357-370

18 Abernethy $V$, Grunebaum $H$. Toward a family planning program in psychiatric hospitals. Am J Public Health 1972; 62: 1638-1646
19 Puente F. Family planning for psychiatric patients. IPPF Med Bull 1977; 11: $2-4$

20 Muhuhu P. Family planning: an integral part of mental health care. J Famil Health Train 1982; 1: 7-9

21 Kölch M, Schmid M. Elterliche Belastung und Einstellungen zur Jugendhilfe bei psychisch kranken Eltern: Auswirkungen auf die Inanspruchnahme von Hilfen. Prax Kinderpsychol Kinderpsychiatr 2008; 57: 744-788

22 Seidel $R$. Erinnern oder vergessen? Der schwierige Neubeginn. Die deutsche Psychiatrie nach 1945 im Schatten der nationalsozialistischen Patientenmorde. Soziale Psychiatrie 2000; 3: 39-43

23 Könnecke R, Wening $U$, Ropeter $D$ et al. Sozialer Entwicklungsstand und subjektives Belastungserleben bei Nachkommen Schizophrener. Qualitative und quantitative Ergebnisse aus der Mannheimer Risikokinderstudie. Psychiat Prax 2006; 33: 269-276

24 Jungbauer J, Stelling K, Kuhn J et al. Wie erleben schizophren erkrankte Mütter und Väter ihre Elternschaft? Ergebnisse einer qualitativen Interviewstudie. Psychiat Prax 2010; 37: 233-239 